BMJ Open

Diabetes

Research

\& Care

\title{
Reduced circulating levels of chemokine CXCL14 in adolescent girls with polycystic ovary syndrome: normalization after insulin sensitization
}

\author{
Cristina García-Beltran, ${ }^{1,2}$ Ruben Cereijo, ${ }^{3,4}$ Tania Quesada-López, ${ }^{3,4}$ \\ Rita Malpique, ${ }^{1}$ Abel López-Bermejo, ${ }^{5,6}$ Francis de Zegher, ${ }^{7}$ Lourdes Ibáñez, ${ }^{1,2}$ \\ Francesc Villarroya (D) ${ }^{3,4}$
}

To cite: García-Beltran C Cereijo R, Quesada-López T, et al. Reduced circulating levels of chemokine CXCL14 in adolescent girls with polycystic ovary syndrome: normalization after insulin sensitization. BMJ Open Diab Res Care 2020;8:e001035. doi:10.1136/ bmjdrc-2019-001035

- Additional material is published online only. To view please visit the journal online (http://dx.doi.org/10.1136/ bmjdrc-2019-001035).

CG-B and RC are joint first authors.

$\mathrm{LI}$ and FV are joint last authors.

Received 11 November 2019 Revised 20 January 2020 Accepted 29 January 2020

Check for updates

C Author(s) (or their employer(s)) 2020. Re-use permitted under CC BY-NC. No commercial re-use. See rights and permissions. Published by BMJ.

For numbered affiliations see end of article.

Correspondence to Dr Francesc Villarroya; fvillarroya@ub.edu

\section{ABSTRACT}

Objective CXCL14 (C-X-C motif chemokine ligand-14) is a chemokine released by active brown fat, showing protective effects against insulin resistance in experimental models. Polycystic ovary syndrome (PCOS) in adolescent girls is usually related to hepato-visceral fat excess and insulin resistance, and associates with comorbidities such as type 2 diabetes. Treatment with a low-dose combination of one antiandrogen and antimineralocorticoid drug (spironolactone) and two insulin sensitizers (pioglitazone/ metformin) (SPIOMET) is particularly effective in improving these metabolic derangements. Adipose tissue may be involved in the metabolic alterations of PCOS, and it is a likely target of therapeutic action. We investigated the alterations in CXCL14 levels and the effects of drugs composing SPIOMET treatment on CXCL14 in human adipocytes.

Research design and methods We studied 51 adolescent patients with PCOS and 21 age-matched healthy controls. Thirty-one adolescent patients with PCOS under SPIOMET or oral contraception-based treatment were also studied. For studies in vitro, Simpson Golabi Behmel Syndrome (SGBS) adipose cells were used. Gene expression for CXCL14 and other genes was quantified using quantitative real-time PCR. The levels of CXCL14 and adipokines in serum and cell culture media were determined by ELISA.

Results Serum CXCL14 levels are reduced in patients with PCOS. One-year SPIOMET treatment normalized CXCL14 concentrations and improved the metabolic status of patients with PCOS. Pioglitazone induced CXCL14 expression in differentiating human SGBS adipocytes, in parallel with the induction of marker genes of brown adipogenesis. Spironolactone induced CXCL14 expression and release in differentiated human adipocytes. Conclusion Insulin sensitization with SPIOMET normalizes the abnormally low levels of CXCL14 in girls with PCOS. This is consistent with the effects of pioglitazone and spironolactone inducing CXCL14 expression and promoting a brown-like phenotype in adipocytes. CXCL14 may be a novel biomarker for PCOS as well as a potential mediator of the beneficial effects of the SPIOMET combination and may hold promise as a therapeutic modulator of the disorder. Trial registration numbers ISRCTN29234515 and ISCRCTN11062950.

\section{Significance of this study}

What is already known about this subject?

- Treatment with a low-dose combination of one mixed antiandrogen and antimineralocorticoid (spironolactone), and two insulin sensitizers (pioglitazone/metformin) (SPIOMET) is particularly effective in improving the endocrine-metabolic derangements in adolescent girls with polycystic ovary syndrome (PCOS).

- CXCL14 (C-X-C motif chemokine ligand-14) is a chemokine released by active brown fat and protective against insulin resistance in experimental models.

What are the new findings?

- Serum CXCL14 levels are abnormally reduced in patients with PCOS.

- SPIOMET treatment for 1 year normalized CXCL14 concentrations and improved the endocrine-metabolic status of patients with PCOS.

- Pioglitazone and spironolactone, drug components of SPIOMET, induce CXCL14 expression and release in human adipocytes, in parallel with the induction of marker genes of brown adipogenesis.

How might these results change the focus of research or clinical practice?

- CXCL14 may become a novel biomarker for PCOS. In addition, CXCL14 appears as a potential mediator of the beneficial effects of the SPIOMET combination and may hold the capacity of serving as therapeutic modulator of the disorder.

\section{INTRODUCTION}

Adipose tissue plasticity is growingly recognized as a relevant factor for the development of metabolic syndrome, independently of the presence or absence of obesity. The acquisition of a 'brown' or 'beige' phenotype by adipose tissue is considered protective against hyperglycemia and hyperlipidemia and, indeed, the relative protection against these alterations 
in young versus elder individuals is associated with the known prevalence of the brown/beige phenotype in early human development. ${ }^{1}$ CXCL14 (C-X-C motif chemokine ligand-14) is a chemokine produced by active brown/beige adipose tissue, capable of improving glucose metabolism in insulin-resistant rodent models. ${ }^{2}$

Central (hepato-visceral) fat excess and insulin resistance are common metabolic comorbidities in girls and young women with polycystic ovary syndrome (PCOS). ${ }^{34}$ Treatment with a low-dose combination of one mixed antiandrogen and antimineralocorticoid (spironolactone) and two insulin sensitizers (pioglitazone plus metformin) (SPIOMET) has been shown to improve the metabolic condition of these patients to a better extent than oral contraception (OC). ${ }^{5}$ However, the specific cellular and tissue targeting, and the relative role of each of the SPIOMET components in the context of PCOS is poorly understood. It has been recently reported that women with PCOS have lower brown adipose tissue (BAT) activity as compared with healthy controls, ${ }^{67}$ and experimental data suggest that BAT activation might be a promising therapeutic option for PCOS. ${ }^{8}$

Here, we report abnormally low levels of CXCL14 in girls with PCOS, the differential effects of SPIOMET and OC on circulating CXCL14 in relation to metabolic improvement, and the effects of SPIOMET components on the expression and release of CXCL14 in a cellular model of human adipocytes.

\section{RESEARCH DESIGN AND METHODS}

\section{Study population and design}

The study population consisted of 52 adolescent girls with PCOS (age, 15.6years; body mass index (BMI), $24.3 \mathrm{~kg} / \mathrm{m}^{2}$ ) who were enrolled into two randomized, open-label, controlled trials (with identical design) exploring the effects of OC versus SPIOMET treatment for 1 year, with post-treatment ovulation rate as primary outcome. The results of the first trial (ISRCTN29234515) have been already published, ${ }^{5}$ and the second trial (ISCRCTN11062950) will be completed in 2019. Twenty-one out of the 52 girls belonged to the first study and 31 to the second trial; 31 of the 52 had available samples for CXCL14 assessment at 0 and 12 months (online supplementary figure 1). Both trials were performed at Sant Joan de Déu University Hospital, Barcelona, Spain. Inclusion and exclusion criteria have been described previously. ${ }^{5}$ OC treatment consisted of $20 \mu \mathrm{g}$ of ethinylestradiol plus $100 \mathrm{mg}$ of levonorgestrel for 21/28 days and placebo for 7/28 days; SPIOMET is a low-dose combination of spironolactone $50 \mathrm{mg} /$ day, pioglitazone $7.5 \mathrm{mg} /$ day, and metformin $850 \mathrm{mg} /$ day. Twenty-one agematched healthy girls recruited in nearby schools served as controls. All had regular menstrual cycles and none was hirsute or taking medications.

\section{Clinical, endocrine-metabolic, and imaging assessments}

Birth weight and BMI (and their Z-scores) were retrieved, and endocrine-metabolic variables were assessed in the early morning, in the follicular phase (days 3-7) of the cycle, or after 2 months of amenorrhea ${ }^{59}$ Serum glucose, insulin, homeostasis model assessment-insulin resistance (HOMA-IR), lipids, ultrasensitive $\mathrm{C}$ reactive protein (usCRP), sex hormone-binding globulin, androgens, and high-molecular-weight (HMW) adiponectin were assessed as reported. ${ }^{5}$ CXCL14 levels in serum and cell culture medium were determined using a specific ELISA kit (RayBiotech), ${ }^{2}$ whose sensitivity was $0.7 \mathrm{ng} / \mathrm{mL}$, and interassay and intra-assay coefficients of variation less than $12 \%$.

Body composition was assessed by dual X-ray absorptiometry with a Lunar Prodigy and Lunar software (version 3.4/3.5; Lunar, Madison, Wisconsin, USA); abdominal (subcutaneous and visceral) and hepatic fat were assessed by MRI using a multiple-slice MRI 1.5 Tesla scan (Signa LX Echo Speed Plus Excite; General Electric, Milwaukee, Wisconsin, USA). ${ }^{5}$ Central fat was arbitrarily defined as the sum of visceral fat (in squared centimeter) and hepatic fat (in per cent).

\section{Studies in human adipocytes in culture}

The effects of pioglitazone, spironolactone, and metformin on adipocytes were studied in human Simpson Golabi Behmel Syndrome (SGBS) cells, a cell model of human beige (brown-like) adipogenesis. ${ }^{10}{ }^{11}$ SGBS preadipocytes were maintained in Dulbecco's modified Eagle's (DMEM)/F12 medium, 10\% FBS. Adipogenic differentiation was initiated by incubating confluent cell cultures for 4 days in serum-free medium plus $20 \mathrm{nM}$ insulin, $0.2 \mathrm{nM}$ triiodothyronine, $100 \mathrm{nM}$ cortisol, $25 \mathrm{nM}$ dexamethasone, $500 \mu \mathrm{M}$ 3-isobutyl-1-methyl-xanthine, and $2 \mu \mathrm{M}$ rosiglitazone. Subsequently, the cells were switched to DMEM/F12, $20 \mathrm{nM}$ insulin, $0.2 \mathrm{nM}$ triiodothyronine, and $100 \mathrm{nM}$ cortisol and maintained for up to 10 days, when more than $90 \%$ cells have acquired differentiated adipocyte morphology. Two experimental designs were followed: (1) cells were treated with the medications across their differentiation process, in the absence of rosiglitazone and maintaining the drugs throughout the 10 days of differentiation and (2) adipocytes were treated with the drugs acutely (24hours), once the cells have been differentiated. Controls included a 1:1 methanol/ dimethyl sulfoxide mixture (drug solvents) at $\leq 1 / 1000$ concentration. Cell culture reagents and drugs were from Sigma. The cell culture medium was collected and concentrated 1:5 prior to measurement of CXCL14 levels. RNA was extracted from cells using an affinity columnbased method (Machery-Nagel). Real-time quantitative reverse transcription PCRs were performed using $0.5 \mu \mathrm{g}$ RNA and employing TaqMan reagents and probes (Life Technologies), according to supplier indications. PCR was conducted in an ABI/Prism-7700 Sequence Detector System. The following TaqMan probes were used: CXCL14, Hs01557413; uncoupling protein-1 (UCP1), Hs00222453; fatty acid-binding protein-4 (FABP4), Hs00609791, and ribosomal protein lateral stalk subunit 0 (RPLP0) mRNA, Hs99999902. Each sample was run in duplicate and the mean value was used to calculate the 
relative amount of individual mRNAs. Each mean value was normalized to that of the RPLP0 mRNA using the comparative (2- $\Delta \mathrm{CT})$ method.

\section{Statistical analysis and ethics}

Statistical analyses were performed with SPSS V.23.0 (SPSS, Chicago, Illinois, USA). Baseline differences in CXCL14 concentrations between patients and controls were tested with unpaired t-test; covariance analysis was used to adjust for age and BMI. Longitudinal changes between groups were compared by repeated-measures general linear model. Differences in longitudinal changes between groups were tested by the interaction term among between- and within-subject effects. Associations were sought by Pearson correlation analysis. $\mathrm{P}$ value $<0.05$ was considered statistically significant. Results are expressed as mean \pm SD.

The study was conducted after approval by the Institutional Review Board of Sant Joan de Déu University Hospital, after written consent by the parents and assent by each of the participants.

\section{RESULTS}

\section{Serum CXCL14 levels are reduced in adolescent girls with} PCOS

At baseline, PCOS girls showed lower sex hormone-binding protein (SHGB) levels, higher total testosterone concentrations and free androgen index, increased central and hepatic fat, and a trend toward higher insulin and lower HMW adiponectin levels versus controls, as expected (online supplementary table 1 ). None of the girls were obese (BMI $\geq 30 \mathrm{~kg} / \mathrm{m}^{2}$ ); however, mean BMI was higher in girls with PCOS as compared with control girls $(p=0.01)$. Fasting glucose levels were within the normal range in both subgroups and were marginally higher in control girls (online supplementary table 1). Serum CXCL14 concentrations were reduced in girls with PCOS close to twothirds of the normal levels (figure 1A), did not correlate with markers of adiposity or glucose homeostasis, and were positively associated with HMW adiponectin levels (online supplementary table 2 ).

Serum CXCL14 concentrations were reduced in girls with PCOS close to two-thirds of the normal levels (figure 1A) and were positively associated with HMW adiponectin levels (online supplementary table 2). CXCL14 did not correlate with other markers of adiposity or glucose homeostasis; however, there was a trend toward a positive correlation with circulating glucose and a negative correlation with hepatic fat (online supplementary table 2 ).

\section{SPIOMET, but not OC, normalizes serum CXCL14 levels in adolescent girls with PCOS}

SPIOMET treatment was associated with more benefits than OC on endocrine-metabolic and imaging markers, including on fasting insulin, HOMA-IR, HMW adiponectin, usCRP, and hepato-visceral (central) fat, consistent with previous observations ${ }^{5}$ (online supplementary table 3). Serum CXCL14 levels increased significantly after SPIOMET, reaching levels similar to those in
A

B
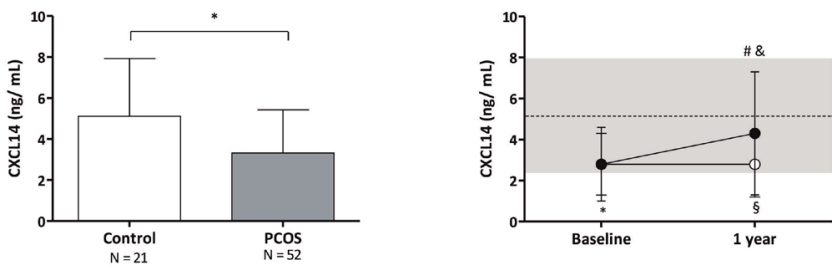

Figure 1 (A) Baseline circulating CXCL14 (C-X-C motif chemokine ligand-14) concentrations in girls with polycystic ovary syndrome (PCOS, $n=52$ ) and in healthy age-matched controls $(n=21)$. Data are mean $\pm S D$. ${ }^{*} P=0.007$, for patients versus controls at baseline. (B) Longitudinal results of CXCL14 serum concentrations in girls with PCOS who received an oral contraceptive (OC, white circles, $n=16$ ) or low-dose spironolactone, pioglitazone, and metformin (SPIOMET, black circles, $n=15$ ). The dotted line is the mean in healthy controls $(n=21)$; the shaded area represents the mean $\pm S D$ in those healthy controls. ${ }^{*} P=0.004$, for patients versus controls at baseline; $\$ p=0.02$, on treatment differences between controls and the OC subgroup; \#p=0.01, for changes $0-1$ year in the SPIOMET subgroup; \&p=0.04 for changes 0-1 year between the OC subgroup and the SPIOMET subgroup.

controls and remained significantly decreased after OC, as compared with controls and with SPIOMET-treated girls (figure 1B). The increase in CXCL14 levels in the SPIOMET-treated patients correlated significantly with the extent of reduction in fasting insulin and HOMA-IR (online supplementary table 4 ).

\section{Effects of the SPIOMET components on CXCL14 expression and release by human adipocytes}

The effects of the three components of SPIOMET on human adipocyte differentiation were assessed individually and in combination (figure 2). Pioglitazone had a doseresponse dramatic effect inducing CXCL14 mRNA expression, whereas neither spironolactone nor metformin did. No concentration higher than $10 \mu \mathrm{M}$ could be tested for spironolactone, because of cell toxicity on preadipocytes. Indeed, pioglitazone at the lowest concentration tested $(0.1 \mu \mathrm{M})$ already caused a close to 10 -fold induction of CXCL14 mRNA expression (figure 2A). This effect paralleled a strong positive effect of pioglitazone on adipogenic differentiation, as evidenced by lipid droplet accumulation (figure 2C) and expression of gene markers of general adipogenic (FABP4) and beige adipogenic (UCP1) differentiation (figure 2D).

Addition of metformin to pioglitazone did not modify the extent of pioglitazone induction of CXCL14 mRNA expression (figure 2B). However, the addition of spironolactone reduced significantly the effects of pioglitazone on CXCL14 mRNA expression. The induction of CXCL14 mRNA expression caused by the three drugs in combination was similar to that elicited by pioglitazone plus spironolactone. These data also paralleled the observations on adipogenic differentiation after combined treatments, according to cell morphology and expression of FABP4 
A

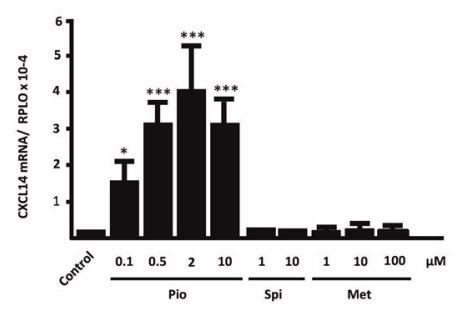

C

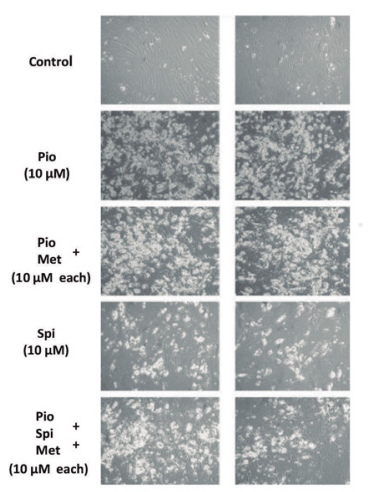

B

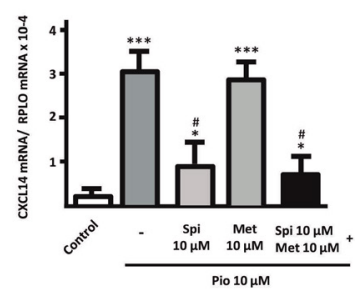

D
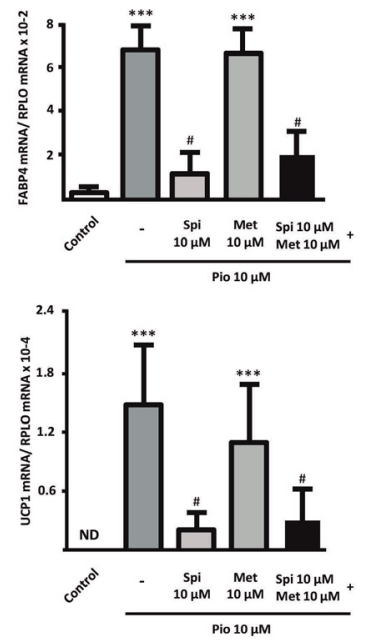

Figure 2 Effects of pioglitazone (Pio), spironolactone (Spi), and metformin (Met) on adipogenic differentiation of SGBS human preadipocytes in culture. SGBS human preadipocytes were treated chronically (10 days) with pioglitazone, spironolactone, or metformin alone $(A)$ or in combination $(B, C, D)$ at the indicated doses across the adipogenic differentiation process. (A, B) CXCL14 (C-X-C motif chemokine ligand-14) transcript levels are presented as means $\pm S D$ from four to five independent experiments and are expressed relative to values from untreated control cells. (C) Representative photomicrographs of adipocyte cell cultures differentiating in the presence of the indicated components: top: control; next to top: pioglitazone $(10 \mu \mathrm{M})$; middle: pioglitazone $(10 \mu \mathrm{M})$ and metformin $(10 \mu \mathrm{M})$; next to bottom: spironolactone $(10 \mu \mathrm{M})$; bottom: pioglitazone $(10 \mu \mathrm{M})$, spironolactone $(10 \mu \mathrm{M})$, and metformin $(10 \mu \mathrm{M})$. Each column represents duplicate representative pictures per treatment condition. (D) Fatty acid-binding protein-4 (FABP4) and uncoupling protein-1 (UCP1) transcript levels are presented as means \pm SD from three to four independent experiments and are expressed relative to values from untreated control cells. ${ }^{\star} P<0.05,{ }^{* \star} p<0.01$, and ${ }^{\star \star *} p<0.001$ for each component versus control; ${ }^{*} p<0.05$ relative to pioglitazone alone. ND, not detected; SGBS, Simpson Golabi Behmel Syndrome.

and UCP1 (figure 2C,D). CXCL14 protein was only detectable in the cell culture medium of differentiating cells under pioglitazone treatment, either alone $(0.185 \mathrm{ng} / \mathrm{mL})$ or in combination with spironolactone and metformin $(0.179 \mathrm{ng} / \mathrm{mL})$.

Neither pioglitazone nor metformin alone had any effect on CXCL14 mRNA expression on already differentiated human adipocytes (figure 3A). However,

A

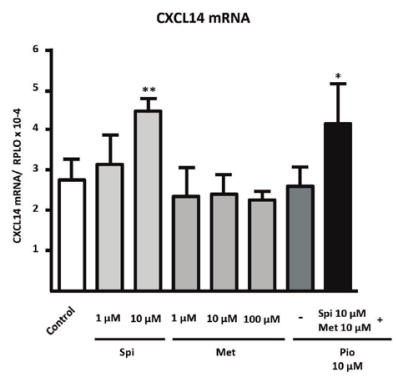

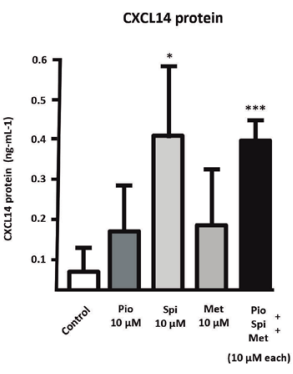

Figure 3 Effects of pioglitazone (Pio), spironolactone (Spi), and metformin (Met) on CXCL14 (C-X-C motif chemokine ligand-14) gene expression and CXCL14 protein release in human adipocytes. SGBS cells were treated acutely (24 hours, at the indicated doses) when already differentiated adipocytes. CXCL14 transcript level (A) and CXCL14 protein levels in cell culture medium (B) are presented as means \pm SD from four to five independent experiments and are expressed relative to values from untreated control cells. ${ }^{*} P<0.05$, ${ }^{* *} \mathrm{p}<0.01$, and ${ }^{* * *} \mathrm{p}<0.001$ for each component versus control. SGBS, Simpson Golabi Behmel Syndrome.

$10 \mu \mathrm{M}$ of spironolactone caused a significant induction of CXCL14 mRNA expression (figure 3A). The combination of three components (with spironolactone dosed at $10 \mu \mathrm{M}$ ) induced CXCL14 mRNA to the same extent as treatment with spironolactone alone. Only spironolactone, either alone or in combination with pioglitazone plus metformin, elicited a significant increase (close to eightfold) of the CXCL14 protein levels released by adipocytes to the cell culture medium (figure 3B).

\section{DISCUSSION}

Our study shows that PCOS associates with reduced levels of CXCL14, a chemokine recently proposed to be secreted preferentially by brown/beige adipose tissue. ${ }^{2}$ The restoration of normal levels of CXCL14 with SPIOMET, the capacity of pioglitazone to increase CXCL14 expression in preadipocytes, and the capacity of spironolactone to increase the release of CXCL14 in adipocytes, respectively, suggest that SPIOMET treatment could target adipose tissue to improve the metabolic profile of patients with PCOS. In addition, the normalization of central fat after SPIOMET treatment may indicate that circulating CXCL14 relates to ectopic fat rather than to BMI per se.

High CXCL14 levels have been shown to improve insulin sensitivity in adipocytes in vitro ${ }^{12}$ and in rodent models ${ }^{2}$; however, not all reports agree in the antidiabetic actions of CXCL14, and even deleterious prodiabetogenic effects have been proposed in vitro ${ }^{13}$ and in vivo. ${ }^{14}$ Our study, which is, to our knowledge, the first exploring CXCL14 in human metabolic pathology, indicates that normalization of CXCL14 after SPIOMET associates with decreased insulin resistance. Pioglitazone increases insulin sensitivity as well as adipogenic differentiation and also promotes the 
acquisition of a brown/beige phenotype in adipose cells. ${ }^{15}$ The induction of CXCL14 expression by pioglitazone is consistent with data in experimental models highlighting increased expression of CXCL14 with brown/beige adipogenic differentiation. ${ }^{2}$ The positive effects of spironolactone-inducing CXCL14 in differentiated adipocytes are also consistent with previous reports indicating that spironolactone induces adipose tissue browning in rodents ${ }^{16}$ and activates brown fat in humans. ${ }^{17}$ Recent reports disclosed impaired BAT activity in PCOS subjects ${ }^{6}$ and the capacity of brown fat activation to ameliorate PCOS in experimental models. ${ }^{8}$ Considering that CXCL14 is preferentially released by brown adipocytes, ${ }^{2}$ it may be speculated that SPIOMET treatment drives a shift in adipose tissue plasticity to a more brown/beige phenotype resulting in enhanced CXCL14 release, potentially improving glucose homeostasis and possibly reducing diabetes risk. This sequence may be especially relevant in young girls, where the brown/beige adipose tissue amount is particularly significant. ${ }^{18}$

Our study has obvious limitations. First, the effect of SPIOMET on CXCL14 levels in tissues other than adipose tissue cannot be ruled out. Second, the physiological significance of the drug concentrations used in the in vitro studies is unclear. The concentrations of pioglitazone increasing CXCL14 levels in differentiated adipocytes (from at least $0.1 \mu \mathrm{M}$ ) were in the range of those in plasma from SPIOMET-treated healthy women. ${ }^{19}$ However, although spironolactone concentrations used here were the same as those employed in previous studies in adipose cells, ${ }^{16}{ }^{20}$ plasma spironolactone levels in SPIOMET-treated controls are lower. ${ }^{19}$ Finally, evidence for a direct role of CXCL14 downregulation and reinduction on the metabolic status of patients with PCOS after SPIOMET is obviously lacking, as it would imply intervention experiments beyond the ethical standards of human studies. Nevertheless, our data support a role for CXCL14 as a novel potential biomarker and molecular mediator in the improvement of PCOS-associated metabolic alterations following SPIOMET intervention.

\section{Author affiliations}

${ }^{1}$ Pediatric Research Institute Hospital Sant Joan de Déu, Barcelona, Spain ${ }^{2}$ CIBER Diabetes y Enfermedades Metabólicas Asociadas, Madrid, Spain ${ }^{3}$ Biochemistry and Molecular Biomedicine, Institute of Biomedicine, University of Barcelona, Barcelona, Spain

${ }^{4}$ CIBER Fisiopatologia de la Obesidad y Nutrición, Madrid, Spain

${ }^{5}$ Pediatric Endocrinology, Dr. Josep Trueta Hospital, Girona, Spain

${ }^{6}$ Girona Institute for Biomedical Research, Girona, Spain

${ }^{7}$ Department of Development \& Regeneration, University of Leuven, Leuven, Belgium

Contributors CG-B, RC, and TQ-L researched data, contributed to data interpretation, and reviewed/edited manuscript. RM and AL-B contributed to data interpretation and reviewed/edited manuscript. FdZ contributed to study design and data interpretation, and reviewed/edited the manuscript. LI contributed to study design and data interpretation, wrote the manuscript, and reviewed/edited manuscript. FV contributed to study design and data interpretation, wrote the manuscript, and reviewed/edited manuscript.
Funding This work was supported by the Ministerio de Ciencia, Innovación y Universidades, Spain, grant numer SAF2017-85722-R; Instituto de Salud Carlos III, Spain, grant numer PI15/01078; and the Fondo Europeo de Desarrollo Regional (FEDER).

Competing interests None declared.

Patient consent for publication Not required.

Provenance and peer review Not commissioned; externally peer reviewed.

Data availability statement All data relevant to the study are included in the article or uploaded as supplementary information.

Open access This is an open access article distributed in accordance with the Creative Commons Attribution Non Commercial (CC BY-NC 4.0) license, which permits others to distribute, remix, adapt, build upon this work non-commercially, and license their derivative works on different terms, provided the original work is properly cited, appropriate credit is given, any changes made indicated, and the use is non-commercial. See: http://creativecommons.org/licenses/by-nc/4.0/.

ORCID iD

Francesc Villarroya http://orcid.org/0000-0003-1266-9142

\section{REFERENCES}

1 Cereijo R, Giralt M, Villarroya F. Thermogenic brown and beige/brite adipogenesis in humans. Ann Med 2015;47:169-77.

2 Cereijo R, Gavaldà-Navarro A, Cairó M, et al. CXCL14, a brown adipokine that mediates brown-fat-to-macrophage communication in thermogenic adaptation. Cell Metab 2018;28:750-63.

3 de Zegher F, López-Bermejo A, Ibáñez L. Central obesity, faster maturation, and 'PCOS' in girls. Trends Endocrinol Metab 2018;29:815-8.

4 Ibáñez L, de Zegher F. Polycystic ovary syndrome in adolescent girls. Pediatr Obes 2020;15:e12586.

5 lbáñez L, Del Río L, Díaz M, et al. Normalizing ovulation rate by preferential reduction of hepato-visceral fat in adolescent girls with polycystic ovary syndrome. J Adolesc Health 2017;61:446-53.

6 Shorakae S, Jona E, de Courten B, et al. Brown adipose tissue thermogenesis in polycystic ovary syndrome. Clin Endocrinol 2019;90:425-32.

7 Flávia R O, Mamede M, Bizzi MF, et al. Brown adipose tissue activity is reduced in women with polycystic ovary syndrome. Eur J Endocrinol 2019;181:473-80.

8 Yuan X, Hu T, Zhao H, et al. Brown adipose tissue transplantation ameliorates polycystic ovary syndrome. Proc Natl Acad Sci U S A 2016;113:2708-13.

9 de Zegher F, Reinehr T, Malpique R, et al. Reduced prenatal weight gain and/or augmented postnatal weight gain precedes polycystic ovary syndrome in adolescent girls. Obesity 2017;25:1486-9.

10 Wabitsch M, Brenner RE, Melzner I, et al. Characterization of a human preadipocyte cell strain with high capacity for adipose differentiation. Int J Obes Relat Metab Disord 2001;25:8-15.

11 Yeo CR, Agrawal M, Hoon S, et al. SGBS cells as a model of human adipocyte browning: a comprehensive comparative study with primary human white subcutaneous adipocytes. Sci Rep 2017;7:4031.

12 Takahashi M, Takahashi Y, Takahashi K, et al. CXCL14 enhances insulin-dependent glucose uptake in adipocytes and is related to high-fat diet-induced obesity. Biochem Biophys Res Commun 2007;364:1037-42.

13 Atanes P, Hawkes RG, Olaniru OE, et al. CXCL14 inhibits insulin secretion independently of CXCR4 or CXCR7 receptor activation or cAMP inhibition. Cell Physiol Biochem 2019;52:879-92.

14 Nara N, Nakayama Y, Okamoto S, et al. Disruption of CXC motif chemokine ligand-14 in mice ameliorates obesity-induced insulin resistance. J Biol Chem 2007;282:30794-803.

15 Foellmi-Adams LA, Wyse BM, Herron D, et al. Induction of uncoupling protein in brown adipose tissue. synergy between norepinephrine and pioglitazone, an insulin-sensitizing agent. Biochem Pharmacol 1996;52:693-701.

16 Armani A, Cinti F, Marzolla V, et al. Mineralocorticoid receptor antagonism induces browning of white adipose tissue through impairment of autophagy and prevents adipocyte dysfunction in high-fat-diet-fed mice. Faseb J 2014;28:3745-57.

17 Thuzar M, Law WP, Dimeski G, et al. Mineralocorticoid antagonism enhances brown adipose tissue function in humans: a randomized placebo-controlled cross-over study. Diabetes Obes Metab 2019;21:509-16. 
18 Gilsanz V, Hu HH, Kajimura S. Relevance of brown adipose tissue in infancy and adolescence. Pediatr Res 2013;73:3-9. 1.

19 Ballester MR, Puntes M, Martínez J, et al. Optimizing the formulation an the study design to assess the comparative bioavailability of a new film-coated tables (combination of Spironolactone $50 \mathrm{mg}$, Pioglitazone $7.5 \mathrm{mg}$, and Metformin $850 \mathrm{mg}$ ) versus the same individual components in healthy women, under fed conditions. 2nd International Conference on Medicinal Chemistry \& Drug Design and 2nd International

Conference on Bio Equivalence \& Bio Availability, 2019.

20 Corbould A. Effects of spironolactone on glucose transport and interleukin- 6 secretion in adipose cells of women. Horm Metab Res 2007;39:915-8. 